\title{
MUSICOTERAPIA EN EL S. XXI EN ESPAÑA: SITUACIÓN Y EVOLUCIÓN PROFESIONAL, FORMATIVA Y ASOCIATIVA
}

\author{
Music therapy in the 21st century in spain: professional, training and associative \\ situation and development
}

Musicoterapia no século XXI na espanha: situação e desenvolvimento profissional, formativo e associativo

Vicente Alejandro March Lujan

Resumen - El desarrollo de la Musicoterapia en España es bastante reciente, introduciéndose la disciplina en los años 70 del s. XX de la mano de la Dra. Serafina Poch Blasco. Desde entonces, se fue consolidando formación en la materia, en primera instancia en cursos y jornadas de sensibilización y como ampliación de recursos asistenciales para profesionales afines, y más tarde en formato de postgrados-maestrías ya en universidades (públicas y privadas) y en centros privados. Al mismo tiempo, los musicoterapeutas han venido organizándose como colectivo a través de distintas agrupaciones, y llevando a cabo iniciativas, por una parte, para el crecimiento intraprofesional; y por otra, acciones de índole política de cara a una regulación profesional que, si bien se antoja cercana, aún no ha llegado. Este artículo pretende realizar un recorrido por los principales eventos acontecidos que en los últimos tiempos han marcado la evolución profesional, formativa y asociativa de la Musicoterapia.

Palabras clave: musicoterapia, siglo XXI, evolución, España.

Abstract - The developmentof Music Therapy in Spainis quite recent, the discipline was introduced in the 70's of the 20th century, by Dr. Serafina Poch Blasco. Since then, training in the field has been consolidated, firstly in courses and conferences to raise awareness and as an extension of care resources for related professionals; and later in the form of post graduate-master's degrees in universities (public and private) and in private centers. At the same time, music therapists have been organizing themselves as a collective through different groups and carrying out initiatives, on the one hand, for intra-professional growth; and on the other, political actions for professional regulation, which, although it seems close, has not yet arrived.This article aims to review the main events that have marked the professional, formative and associative evolution of Music Therapy in recent times.

Keywords: music therapy, XXI century, evolution, Spain.

Resumo - O desenvolvimento da musicoterapia na Espanha é bastante recente, tendo sido a disciplina introduzida, nos anos 70, pela Dra. Serafina Poch Blasco. Desde então,

\footnotetext{
${ }^{1}$ Universidad Católica de Valencia. E-mail: alejandro.march@ucv.es.
} 
a formação na matéria foi consolidada, primeiramente em cursos e jornadas de sensibilização e como ampliação dos recursos de cuidados para profissionais de áreas afins; e, mais tarde, sob a forma de pós-graduações em universidades (públicas e privadas) e em centros privados. Ao mesmo tempo, os musicoterapeutas têm se organizado como um coletivo, através de diferentes grupos e levado a cabo iniciativas, por um lado, para o crescimento intra-profissional; e por outro, ações políticas para a regulamentação profissional que, embora pareça próxima, ainda não chegou. Este artigo pretende trazer os principais eventos que marcaram a evolução profissional, educacional e associativa da musicoterapia nos últimos tempos.

Palavras-chave: musicoterapia, século XXI, evolução, Espanha. 
Infancia y adolescencia de la musicoterapia en españa: 1970-2000

La Dra. Poch fue la primera autora en escribir sobre Musicoterapia en España, formándose en Estados Unidos (EE. UU.) a finales de los años 60 del s. XX. Como pionera en la materia, realizó una amplia tarea de divulgación e investigación en diferentes centros educativos y psiquiátricos.

Durante la década de los setenta del siglo XX, un grupo de profesionales de las áreas de educación y psicología interesados en la Musicoterapia de diferentes regiones de España tomaron contacto con la Dra. Poch para formarse en la materia, para así poder comenzar a implementar los principios de utilización terapéutica de la música en su trabajo con pacientes y alumnos con necesidades educativas especiales. En Madrid en 1975 se organizó el primer curso de Introducción a la Musicoterapia dictado por el Dr. Rolando Benenzon, cuyo éxito e interés llevaron a que en 1977 se organizara en Madrid el I Symposium Nacional de Musicoterapia. Ese mismo año se constituyó oficialmente la Asociación Española de Musicoterapia (AEMT) (Sabbatella, 2004).

Ya entrando en la década de los ochenta del siglo XX, la Dra. Serafina Poch se traslada a Barcelona y funda, en 1983, la Asociación Catalana de Musicoterapia (ACMT). Ese mismo año, por su parte, Aitor Loroño y Patxi del Campo fundan el Centro de Investigación Musicoterapéutica (CIM) en Vitoria-Gasteiz. Al llegar 1986, Patxi del Campo funda, también en Vitoria, el centro privado Escuela de Musicoterapia y Técnicas Grupales (actualmente con el nombre "Instituto Música, Arte y Proceso (IMAP”). En 1987, Aitor Loroño se traslada a Bilbao y funda el Centro de Investigación Musicoterapéutica (MI-CIM). Ambos centros, desde su creación, han venido impartiendo formación en Musicoterapia, siendo las instituciones pioneras en ofrecer este tipo de estudios en España (European Music Therapy Confederation EMTC, 2021).

A principios de la década de los noventa del siglo XX, en 1993, tuvo lugar el VII Congreso Mundial de Musicoterapia en Vitoria-Gasteiz, que supuso un punto álgido de la visibilidad de la Musicoterapia en nuestro país. Este hecho generó un creciente interés por la Musicoterapia tanto en el ámbito profesional como académico, y profesionales de áreas afines con formación en Musicoterapia comenzaron a implementar los principios de la Musicoterapia en su actividad profesional, en las áreas de educación especial y psiquiatría, aunque mayormente en el ámbito privado. Con este germen empezaron a 
organizarse formaciones en musicoterapia, en forma de seminarios introductorios, talleres de sensibilización y jornadas en diferentes entidades diseminadas por la geografía española, en Universidades, Institutos Privados y en Asociaciones de Musicoterapia. Paralelamente, algunas Universidades y Centros Privados estructuraron programas específicos de Formación en Musicoterapia. Pero no fue hasta 1992 cuando llegó la $1^{\text {a }}$ formación específica destinada a formar musicoterapeutas, con una duración ya considerable: el ler curso de postgrado en Musicoterapia de la Universidad de Barcelona, bajo la dirección de la Dra. Poch. A finales de los años 90 del s. XX, se pusieron en marcha diferentes programas de Musicoterapia en instituciones privadas y públicas, en áreas como educación especial, atención de ancianos con demencias, enfermedad de Alzheimer, rehabilitación neurológica y psiquiatría (Sabbatella, 2004).

\section{La madurez temprana de la musicoterapia en España: 2000-2021}

Toda esta labor de difusión y formación realizada a lo largo los años 70, 80 y 90 del s. XX generó, en diferentes puntos del país, una necesidad de los musicoterapeutas por asociarse, constituyendo se paulatinamente asociaciones de Musicoterapia a lo largo de la geografía española. Posteriormente a estos movimientos a grupacionales, mayormente con carácter regional, fue gestándose la necesidad de un tipo de unión superior, que pudiera facilitar un espacio para que los representantes de estas asociaciones de Musicoterapia pudieran empezar a realizar los trámites políticoadministrativos necesarios que el Estado requiriese para la regulación profesional de la Musicoterapia en España.

Sobre la formación en Musicoterapia en este período

Desde el arranque del primer postgrado de Musicoterapia en 1992, los postgrados y másteres en Musicoterapia empezaron a proliferar en España (Escudé y Acanfora, 2015).

Actualmente, la mayor parte de la oferta formativa en musicoterapia sigue los estándares de la World Federation of Music Therapy (WFMT) y la European Music Therapy Confederation (EMTC), que recomiendan incorporar en el currículo formativo 
el trabajo terapéutico personal, las prácticas supervisadas, así como módulos formativos en investigación.

En España, la titulación que habilita para el ejercicio profesional de la musicoterapia es la de postgrado o máster con un mínimo de $60 \mathrm{ECTS}^{2}$, tomando en cuenta el documento que recoge el acuerdo alcanzado por las AEMTA-EMTC ${ }^{3}$ con título "Criterios para ser Musicoterapeuta Profesional en España", refrendado posteriormente por la Federación Española de Asociaciones de Musicoterapia (FEAMT).

La formación en Musicoterapia que cumple los requisitos anteriores (tanto del documento "Criterios para ser Musicoterapeuta Profesional en España puede cursar en números as entidades (entre Universidades públicas y privadas, centros formativos privados). Para acceder a ellas se requiere tener una titulación universitaria previa (diplomatura, licenciatura o grado), procedentes de los ámbitos socio-sanitario y educativo principalmente (March-Luján, 2021).

Además, existen formaciones de especialización post-master como parte de la formación continua del/de la musicoterapeuta, en ramas como Musicoterapia Hospitalaria, Geriatría, Diversidad funcional, dolor crónico; o bien en modelosmétodos-abordajes en musicoterapia específicos de intervención (Bonny Method of Guided Imagery and Music BMGIM, modelo Benenzon, y Abordaje Plurimodal) (March-Luján, 2021).

Panorama profesional: implementación de proyectos de Musicoterapia en España en distintos ámbitos

Desde los años 80 del s. XX se inicia un desarrollo de la práctica clínica de la Musicoterapia en España, materializándose proyectos en los ámbitos comunitario y

\footnotetext{
${ }^{2}$ Siglas en inglés de European Credit Transfer System, o Sistema Europeo de Transferencia de Créditos. Cada crédito de formación equivale a 25 horas lectivas, de las cuales 10 corresponden a horas de clase, y las 15 restantes contemplan el trabajo autónomo que realiza el alumno para integrar el aprendizaje (estudio, lecturas, confección de trabajos individuales o grupales, tutorías, etc).

${ }^{3}$ Asociaciones españolas de Musicoterapia (AEMTA) pertenecientes a Confederación Europea de Musicoterapia (EMTC)
} 
educativo, dirigidos principalmente al trabajo con personas en el campo de la salud mental, discapacidad intelectual y geriatría.

En el ámbito socio-sanitario se han venido desarrollando proyectos de Musicoterapia en servicios hospitalarios tales como neonatología, cirugía pediátrica, unidad de diálisis, unidad infantil de enfermedades infecciosas, psiquiatría infantil, psiquiatría de adultos, neurología, unidades de rehabilitación, medicina interna, daño cerebral, oncología pediátrica y de adultos, cardiología, UCI pediátrica y de adultos, cuidados paliativos, etc. En la actualidad, algunos de los ejemplos vigentes que ofrecen el servicio musicoterapéutico ${ }^{4}$ (de los que se tiene constancia), se pueden observar en esta tabla 1:

\begin{tabular}{|l|l|}
\hline \multicolumn{1}{|c|}{ Comunidad autónoma } & \multicolumn{1}{c|}{ Relación de centros } \\
\hline Andalucía & $\begin{array}{l}\text { Ayuntamiento de Alcalá de Guadaira (Sevilla) } \\
\text { Hospital de Cádiz } \\
\text { Hospital de Jaén } \\
\text { Hospital de Marbella, en Málaga (unidad de neonatología) } \\
\text { Hospital materno Infantil de Málaga } \\
\text { Hospital Nisa de Sevilla } \\
\text { Hospital universitario "Virgen de las Nieves" de Granada } \\
\text { Hospitales Virgen del Rocío de Sevilla (cuidados paliativos pediátricos) }\end{array}$ \\
\hline Aragón & $\begin{array}{l}\text { Red de salud mental Huesca: hospital de día, centro de día, unidad de media } \\
\text { estancia, unidad de larga estancia y unidad de psicogeriatría }\end{array}$ \\
\hline Castilla la Mancha & $\begin{array}{l}\text { Complejo Hospitalario Universitario de Albacete }{ }^{6} \text { CHUA } \\
\text { Castilla- León }\end{array}$ \\
$\begin{array}{l}\text { Centro de Referencia Estatal de Atención a personas con enfermedad de } \\
\text { Alzheimer CREA Salamanca7 } \\
\text { Servicio de Musicoterapia asistencial domiciliaria para pacientes de cuidados }\end{array}$ \\
\hline
\end{tabular}

\footnotetext{
${ }^{4}$ Cabe indicar que en algunos se hace en forma de voluntariado, y otros de manera remunerada. A causa de la pandemia mundial por Covid 19 acontecida en 2020 y 2021, el servicio de Musicoterapia en muchos de estos centros se ha suspendido, si bien en la mayor parte de ellos se está retomando de nuevo.

${ }^{5}$ Gracias al el proyecto SONOS, que se inició en 2005 con la financiación del Gobierno de Aragón, se implantó este programa de Musicoterapia.

${ }^{6}$ Investigación no financiada en la Unidad de Cuidados Intensivos (UCI) con 86 pacientes sobre la influencia de la musicoterapia en la calidad del sueño con pacientes conscientes (despiertos) de esta unidad. Momentáneamente paralizada debido a la situación pandémica por Covid-19. Será retomada en octubre de 2021.

${ }^{7}$ Desde el año 2008, el Centro de Referencia Estatal de Alzheimer cuenta con un musicoterapeuta en plantilla, formando parte del equipo multidisciplinar que atiende tanto a los usuarios del centro como a los cuidadores principales y familiares, en sesiones periódicas semanales, tanto individuales como grupales.
} 


\begin{tabular}{|c|c|}
\hline & paliativos, dependiente del Hospital Universitario de Burgos ${ }^{8}$ \\
\hline Catalunya & $\begin{array}{l}\text { Centre asistencia lEmili Mira (salud mental) } \\
\text { Corporación Sanitaria Parc Taulí } \\
\text { Hospital del Mar (cuidados intensivos) } \\
\text { Hospital Germans Triasi Pujol } \\
\text { Hospital Sant Joan de Déuinfantil } \\
\text { Hospital Sant Pau } \\
\text { Institut Guttman } \\
\text { Parc Sanitari Sant Joan de Déu (área de psiquiatría/discapacidad y cuidados } \\
\text { intensivos) }\end{array}$ \\
\hline $\begin{array}{l}\text { Comunidad Autónoma } \\
\text { de Canarias }{ }^{9}\end{array}$ & $\begin{array}{l}\text { Complejo Hospitalario Universitario de Canarias HUC (Tenerife) } \\
\text { Complejo Hospitalario Universitario Insular Materno Infantil CHUIMI } \\
\text { (Palmas de Gran Canaria) } \\
\text { Complejo Hospitalario Universitario Nuestra Señora de Candelaria HUNSC } \\
\text { (Tenerife) } \\
\text { Hospital Universitario de Gran Canaria Doctor Negrín }{ }^{10}\end{array}$ \\
\hline $\begin{array}{l}\text { Comunidad Foral de } \\
\text { Navarra }\end{array}$ & Hospital San Juan de Dios de Navarra (Unidad de Cuidados paliativos) \\
\hline Comunidad de Madrid & $\begin{array}{l}\text { Hospital } 12 \text { de octubre } \\
\text { Hospital Fundación Instituto San José } \\
\text { Hospital Gregorio Marañón } \\
\text { Hospital La Paz } \\
\text { Hospital Niño Jesús } \\
\text { Hospital Ramón y Cajal } \\
\text { Hospital Universitario de Torrejón } \\
\text { Hospital Universitario Montepríncipe }\end{array}$ \\
\hline Comunitat Valenciana & $\begin{array}{l}\text { CRE de Atención Psicosocial a personas con trastorno mental grave del } \\
\text { IMSERSO (Valencia) }{ }^{11} \\
\text { Hospital "Dr. Moliner" } \\
\text { Hospital "Marina Salud" de Dénia } \\
\text { Hospital "Valencia al Mar" } \\
\text { Hospital psiquiátrico provincial de Bétera (Valencia) }\end{array}$ \\
\hline
\end{tabular}

${ }^{8}$ Proyecto financiado por la obra social de la entidad bancaria "La Caixa".

${ }^{9}$ Desde el año 2016, la Consejería de Sanidad del Gobierno de Canarias firmó un convenio con la Fundación MAPFRE Guanarteme para desarrollar estos proyectos de musicoterapia hospitalaria en todos estos centros, atendiendo a adultos y niños de diversas unidades. En 2019 se atendió a 1609 pacientes. Trabajan un total de 5 musicoterapeutas.

${ }^{10}$ Dentro de este complejo, hospitalario, se realiza musicoterapia dentro del Hospital Juan Carlos I, especializado en atención a la Salud Mental.

${ }^{11}$ Entre sus servicios tiene incorporado un programa de musicoterapia desde el año 2016. Además, inició en 2018 un proyecto específico de I+D+I sobre musicoterapia en salud mental. 


\begin{tabular}{|c|c|}
\hline Extremadura & $\begin{array}{l}\text { Centro socio-sanitario para personas con trastorno mental grave de Plasencia } \\
\text { (Cáceres) } \\
\text { Programa de musicoterapia en geriatría en el tratamiento a pacientes con }^{12} \\
\text { deterioro cognitivo y envejecimiento activo }{ }^{13}\end{array}$ \\
\hline Galicia & $\begin{array}{l}\text { Área sanitaria de Ourense, Verín y Barco de Valdeorras }{ }^{14} \text {. } \\
\text { Hospital Clínico Universitario del Complejo Hospitalario Universitario de } \\
\text { Santiago de Compostela, Área de hospitalización del Servicio de Pediatría } \\
\text { (Unidad de hospitalización de Hematología y Oncología Pediátrica). } \\
\text { Hospital de Día de Psiquiatría del Hospital Universitario de Ourense CHUO }\end{array}$ \\
\hline Illes Balears & $\begin{array}{l}\text { Centro de Día de Personas Mayores, en Maó (Menorca) }{ }^{15} \\
\text { Centro de Día de Promoción de la Autonomía, en Maó (Menorca) }{ }^{16} \\
\text { Centro de Día del Patronato del Hospital Municipal , en Ciutadella } \\
{\text { (Menorca })^{17}} \text {, entro de Día para personas con diversidad funcional, en Maó (Menorca) }{ }^{18} \\
\text { Hospital de Día de Sant Joan de Déu, de Palma de Mallorca (geriatría) }{ }^{19} \\
\text { Hospital Universitario Son Espases, de Palma de Mallorca (atención } \\
\text { domiciliaria en unidad de cuidados paliativos) }{ }^{20} \\
\text { Residencia geriátrica asistida del Patronato del Hospital Municipal, en } \\
\text { Ciutadella (Menorca) }\end{array}$ \\
\hline La Rioja & Hospital General de la Rioja (Unidad de Cuidados paliativos) \\
\hline Navarra & Hospital San Juan de Dios de Navarra (Unidad de Cuidados paliativos) \\
\hline
\end{tabular}

\footnotetext{
${ }^{12}$ Programa subvencionado tanto por la Diputación de Cáceres como por el Gobierno de Extremadura.

${ }^{13}$ Servicio dependiente del Servicio Extremeño de Promoción de la Autonomía y Atención a la Dependencia Gerencia territorial de Badajoz (SEPAD). Atiende por semana a más de 450 pacientes con deterioro cognitivo leve, moderado y demencias avanzadas. En este mismo programa se atiende semanalmente a unos 300 pacientes que no presentan deterioro cognitivo en programas de envejecimiento activo.

${ }^{14}$ Proyecto que se lleva a cabo al firmarse un convenio de colaboración entre el Servicio Galego de Saúde(SERGAS) y Asociación Galega de musicoterapia AGAMUS.

${ }^{15}$ Ídem anterior.

${ }^{16}$ Proyecto dependiente del Consell Insular de Menorca, departamento de Benestar.

17 Ídem anterior.

${ }^{18}$ Ídem anterior.

${ }^{19}$ Subvencionado por Obra Social Sant Joan de Déu

${ }^{20}$ Subvencionado por Obra Social "La Caixa"

${ }^{21}$ Proyecto dependiente del Ajuntament de Ciutadella, con la financiación Obra social Caixa Colonya de Pollença.
} 


\begin{tabular}{|l|l|}
\hline País Vasco & $\begin{array}{l}\text { Hospitales de Organización Sanitaria Integrada OSI Araba } \\
\text { Instituto Foral de Bienestar Social, Álava }\end{array}$ \\
\hline Región de Murcia & $\begin{array}{l}\text { Instituto Murciano de Acción Social (IMAS) (atención a personas con } \\
\text { discapacidad/ personas mayores en residencias) }\end{array}$ \\
\hline
\end{tabular}

Tabla 1 Relación centros socio-sanitarios españoles que ofrecen servicios de musicoterapia actualmente, distribuidos por Comunidades Autónomas ${ }^{24}$ en orden alfabético.

Nota. Información recabada a través de la comunicación con musicoterapeutas con un papel representativo en las distintas Comunidades Autónomas.

Otro ámbito a destacar, por el desarrollo habido de la Musicoterapia en los últimos tiempos, es el educativo. Son muchas las asociaciones y centros escolares que cuentan con sesiones semanales de Musicoterapia para atender a niños, adolescentes y adultos con diversidad funcional y otras patologías. Algunos ejemplos destacables:

- Comunidad Valenciana: desde el año 2013, los centros públicos de educación especial de titularidad de la Generalitat Valenciana disponen de profesorado especialista de Educación Física y de Educación Musical, tomando en cuenta lo que se recoge en el artículo 5 de la Orden 12/2013 de 14 de marzo de la Conselleria de Cultura, Educación y Deporte. Con este decreto, si bien se crea la plaza de maestro especialista en Educación Musical, la dirección de muchos centros ha optado por la contratación de maestros con doble perfil, maestro de música y musicoterapeuta, con la finalidad de atender así a toda la diversidad presente en sus centros.

- Vitoria (País Vasco): la asociación Autismo Araba tiene desde el año 2005 un convenio con el Instituto Música, Arte y Proceso, y gracias a este programa los usuarios de esta asociación cuentan con un programa de musicoterapia donde se atiende a más de 50 niños, adolescentes y jóvenes semanalmente, en sesiones individuales y grupales.

- Pamplona (Navarra): En el Centro de Atención a la Discapacidad Valle del Roncal, la Fundación MAPFRE-Quavitae, junto a la asociación de familiares del

\footnotetext{
${ }^{22}$ En este centro, en convenio con el Gobierno Vasco, se ha iniciado en 2020 la primera investigación en España de musicoterapia en cuidados intensivos en pacientes con ventilación mecánica.

${ }^{23}$ Programa dependiente de la Diputación de Álava. Desde hace 20 que esta institución viene confiando en la Musicoterapia dentro de sus programas sociales, y desde el 2015 cuenta con un programa específico de Musicoterapia, con un equipo de musicoterapeutas que atiende semanalmente a los usuarios de toda la red de residencias y centros de día públicos de la provincia de Álava.

${ }^{24}$ No se ha podido acceder a información de Asturias, Cantabria, y Ciudades Autónomas de Ceuta y Melilla.
} 
centro, financian el programa de musicoterapia. Desde el año 2009 atiende a los más de 100 usuarios residentes del centro en sesiones individuales y/o grupales, en función de sus necesidades. (Grupo de Trabajo para la Regulación de la Musicoterapia en España, 2020)

Por último, dentro del ámbito socio-comunitario existen programas de musicoterapia de largo recorrido en asociaciones e instituciones dentro de los servicios sociales (desprotección infantil, cooperación y atención a la migración, adicciones, exclusión social) y/o instituciones penitenciarias. Algunos ejemplos:

- Programa de intervención musicoterapéutica con migrantes usuarios del centro de día «Algara» de Cruz Roja (San Sebastián, País Vasco).

- Centro de desintoxicación para el tratamiento de las adicciones IVANE, unidad de psicología-psiquiatría (Valencia, Comunidad Valenciana).

- Musicoterapia comunitaria en la cárcel de mujeres de Alcalá de Guadaira (Sevilla, Andalucía) (March-Luján, 2021).

En todos los proyectos, la figura del musicoterapeuta se ha incorporado $y$ continúa incorporando a los equipos interdisciplinares como un profesional más cuya función complementaria resulta plenamente integrada en el equipo.

Para finalizar este apartado sobre la proyección profesional de la Musicoterapia, es necesario destacar que cada vez está más presente en agrupaciones profesionales de otras disciplinas. Como ejemplo, en la Sociedad Española de Cuidados Paliativos (SECPAL) hay un grupo de trabajo denominado MUSPAL (Musicoterapia en cuidados paliativos) que ha elaborado un manual de buenas prácticas de Musicoterapia en Cuidados Paliativos o enfermedades avanzadas. Por otro lado, en la Sociedad española de Geriatría y Gerontología (SEGG) hay socios activos que son Musicoterapeutas y están en vías de creación de un grupo de trabajo propio (Grupo de Trabajo para la Regulación de la Musicoterapia en España, 2020).

Acciones para la regulación profesional de la Musicoterapia en España Entidades representativas de musicoterapeutas profesionales 
Desde finales del S. XX y principios del XXI, varios responsables de centros formativos en Musicoterapia y presidentes-representantes de asociaciones comenzaron a proponer encuentros donde crear las bases de una entidad reguladora de la profesión a nivel nacional. A falta de colegios profesionales ${ }^{25}$, los profesionales de la Musicoterapia se empezaron a organizar, consolidándose diferentes estructuras que velaran por el correcto funcionamiento de la disciplina. Las principales son (por orden de creación): AEMTA-EMTC (Asociación españolas de musicoterapia miembros de la Confederación Europea de Musicoterapia), AEMP (Asociación Española de Musicoterapia) y FEAMT (Federación Española de Asociaciones de Musicoterapia). A continuación, se dará detalle de cada una de ellas.

En el seno de las AEMTA-EMTC surge el cargo de delegado europeo en la EMTC, que es renovado cada 3 años ${ }^{26}$. Pueden pertenecer a ella las asociaciones de Musicoterapia del Estado Español que lo soliciten a la EMTC, registradas en el Ministerio del Interior o del Ministerio de Trabajo. Actualmente, pertenecen a las AEMTA-EMTC (por orden alfabético):

1. Asociación Aragonesa de Musicoterapia AAMT (con sede en Zaragoza, dentro de la Comunidad de Aragón). Su presidenta actual es la musicoterapeuta Da Virtudes Morcillo.

2. Asociación Castellano Leonesa para el Estudio, el Desarrollo y la Investigación de la Musicoterapia y la Arte terapia ACLEDIMA (con sede en Salamanca, dentro de la Comunidad de Castilla- León).Su presidente actual es el musicoterapeuta y Dr.Luis Alberto Mateos.

3. Asociación Catalana de Musicoterapia ACMT (con sede en Barcelona, dentro de la Comunidad de Catalunya). Su presidente actual es el musicoterapeuta D. Pau Gimeno.

\footnotetext{
${ }^{25}$ En la actualidad, solamente se ha conseguido en la Región de Murcia (desde octubre 2017) que esté la Musicoterapia dentro de un colegio profesional. En este caso, existe una sección de musicoterapia dentro del "Colegio de Doctores y Licenciados en Filosofía y Letras de la Región de Murcia". Su inclusión debe considerarse como algo testimonial, pues la Musicoterapia en España no tiene todavía una regulación profesional y, por tanto, esta sección no puede brindar a los profesionales musicoterapeutas un respaldo jurídico pleno, como sucede con otras profesiones reguladas. Pueden consultarse sus estatutos y perfil profesional del musicoterapeuta en https://cdlmurcia.es/estatutos-de-la-seccion-de-musicoterapia/
}

${ }^{26}$ En este caso, la última renovación fue en noviembre de 2019, por lo que estará vigente hasta noviembre 2022. La actual delegada europea es la Dra. Catherine Clancy. Sus funciones vienen detalladas en el documento "Funciones del Delegado/a Español en la European Music Therapy Confederation EMTC" 
4. Asociación para el Desarrollo y la Investigación de la Musicoterapia ADIMTE (con sede en Madrid, si bien tiene carácter estatal). Su presidente actual es el musicoterapeuta y Dr. Alberto Aceves.

5. Asociación Española de Musicoterapeutas profesionales AEMP, organización sindical (con sede en Salamanca, dentro de la Comunidad de Castilla- León, si bien tiene carácter estatal). Su secretario general actual (dado que es un sindicato, no hay presidencia) es el musicoterapeuta y Dr. Luis Alberto Mateos.

6. Asociación Gallega de Musicoterapia AGAMUS (con sede Santiago de Compostela, dentro de la Comunidad de Galicia). Su presidenta actual es la musicoterapeuta $\mathrm{D}^{\mathrm{a}}$ Montserrat López.

7. Asociación Gaditana de Musicoterapia AGAMUT (Cádiz, dentro de la Comunidad de Andalucía).Su presidenta actual es la musicoterapeuta y Dra. $\mathrm{D}^{\mathrm{a}}$ Patricia Sabbatella.

8. Asociación de Profesionales de la Musicoterapia APM (con sede Santiago de Compostela, dentro de la Comunidad de Galicia, si bien tiene carácter estatal). Su presidenta actual es la musicoterapeuta $\mathrm{D}^{\mathrm{a}}$ Montserrat López.

9. Asociación Valenciana de Musicoterapia AVMT (con sede en Valencia, dentro de la Comunidad Valenciana). Su presidenta actual es la musicoterapeuta $\mathrm{D}^{\mathrm{a}}$ Cristina López Gómez

10. Música Arte y Proceso MAP (con sede en Vitoria, dentro del País Vasco). Su presidente actual es el musicoterapeuta D. Patxi del Campo San Vicente (MarchLuján, 2021).

Respecto al trabajo por el desarrollo de la profesión, se hace necesario mencionar las contribuciones realizadas desde el grupo de trabajo de las AEMTAEMTC y por las Subcomisiones de la AEMP. En este sentido, cabe destacar la elaboración de una serie de Documentos Técnicos entre el año 2007 y 2017, realizados tomando en cuenta las directrices dadas por el Espacio Europeo de Educación Superior, el proceso de Convergencia Europea en el marco de titulaciones y acreditación profesional y el Registro Europeo de Musicoterapeutas (European Music Therapy Register, EMTR-EMTC). Estos documentos son (por orden de elaboración): 
- Funciones del Delegado/a Español en la European Music Therapy Confederation (EMTC)(2007, revisión 2017).

- Documento Técnico Recomendaciones Orientativas para Valorar el Diseño de los Postgrados Universitarios de Musicoterapia en España (2008).

- Criterios para ser Musicoterapeuta Profesional en España (2009).

- Registro Español de Musicoterapeutas Acreditados (REMTA, 2010) - Sistema Nacional de Acreditación de Musicoterapeutas - Reglamento de Funcionamiento Interno (RFI) de la Comisión de Acreditación Española de Musicoterapeutas Profesionales (CAEMT, 2010).

- Código Ético de la Musicoterapia en España (2014)

- Criterios para la Organización de Congresos Nacionales de Musicoterapia (2015) (Sabbatella et al., 2018).

Merece una mención especial el REMTA, un proceso expuesto por los agentes de mediación con la Administración Estatal del Ministerio de trabajo como necesario de cara a la regulación profesional futura de la Musicoterapia, según las reuniones que mantuvo el sindicato AEMP con ellos. Dentro de este proceso de registro y acreditación de musicoterapeutas, se establecieron dos categorías, a saber: Musicoterapeuta Acreditado en España (MTAE), en cuya categoría actualmente figuran 94 musicoterapeutas registrados ${ }^{27}$; y Supervisor de Musicoterapia Acreditado en España (SMTAE), en cuya categoría actualmente figuran 26 musicoterapeutas registrados ${ }^{28}$ (AEMP, 2021a). Los requisitos para poder optar a esta categoría de registro pueden apreciarse en la siguiente tabla:

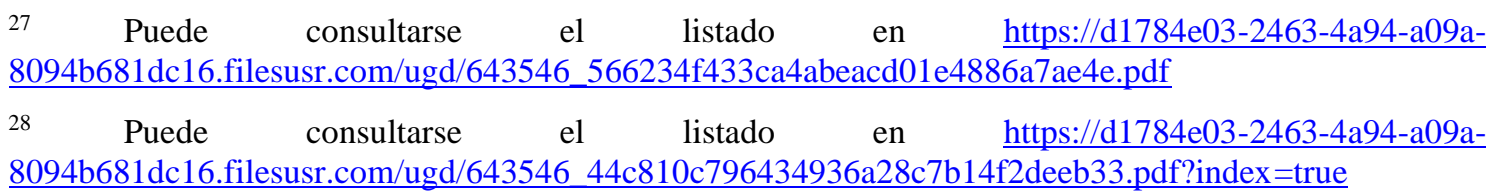




\begin{tabular}{|c|c|}
\hline \multicolumn{2}{|r|}{$\begin{array}{l}\text { REGISTRO ESPAÑOL DE } \\
\text { MUSICOTERAPEUTAS (REMTA) }\end{array}$} \\
\hline CATEGORÍA & $\begin{array}{l}\text { REQUISITOS } \\
\end{array}$ \\
\hline \multirow{6}{*}{$\begin{array}{l}\text { MTAE } \\
\text { Musicoterapeuta } \\
\text { Acreditado en } \\
\text { España }\end{array}$} & $\begin{array}{l}\text { a.- Título de Musicoterapeuta según lo establecido en el criterio } 1 \text { del } \\
\text { Documento Técnico Criterios para ser Musicoterapeuta Profesional } \\
\text { Acreditado en España (MTAE) }\end{array}$ \\
\hline & $\begin{array}{l}\text { b.- Experiencia profesional de un año como musicoterapeuta a tiempo } \\
\text { completo, o equivalente. }\end{array}$ \\
\hline & $\begin{array}{l}\text { c.- Mínimo de } 60 \text { horas de supervisión de práctica clínica. Se podrán } \\
\text { incluir parte de las horas realizadas en la formación inicial, en post- } \\
\text { formación inicial, o de forma mixta. }\end{array}$ \\
\hline & $\begin{array}{l}\text { d.- Mínimo de } 60 \text { horas de proceso formativo didáctico y terapéutico } \\
\text { personal (self-experience): proceso continuo de psicoterapia, } \\
\text { musicoterapia, musicoterapia de grupo, etc.). Se podrán incluir parte } \\
\text { de las horas realizadas en la formación inicial, en post-formación } \\
\text { inicial, o de forma mixta. }\end{array}$ \\
\hline & $\begin{array}{l}\text { e.- Mínimo de } 20 \text { horas de cursos de formación post-formación } \\
\text { inicial y de asistencia a congresos, jornadas u otras actividades } \\
\text { académicas regladas, que estén en relación directa con la } \\
\text { Musicoterapia. }\end{array}$ \\
\hline & $\begin{array}{l}\text { f.- Estar asociado a una Asociación Profesional de Musicoterapia del } \\
\text { ámbito territorial donde se ejerza la profesión y que sea miembro } \\
\text { asociado de la EMTC. }\end{array}$ \\
\hline \multirow{4}{*}{$\begin{array}{l}\text { SMTAE } \\
\text { Supervisor de } \\
\text { Musicoterapia } \\
\text { Acreditado en } \\
\quad \text { España }\end{array}$} & $\begin{array}{l}\text { Podrán optar a ser SMTAE los MTAE que cumplan los siguientes } \\
\text { requisitos: }\end{array}$ \\
\hline & $\begin{array}{l}\text { a.- Experiencia profesional de cinco años como musicoterapeuta a } \\
\text { tiempo completo, o equivalente. }\end{array}$ \\
\hline & b.- Mínimo de 120 horas de supervisión adicionales de práctica clínica. \\
\hline & $\begin{array}{l}\text { c.- Mínimo de } 60 \text { horas de proceso formativo didáctico y } \\
\text { terapéutico personal adicionales (self-experience) }\end{array}$ \\
\hline
\end{tabular}

Tabla 2 Requisitos para ser MTAE y SMTAE según REMTA.

Nota. Adaptado de "Carta $4^{a}$ Convocatoria REMTA 2018", por Comisión Acreditación Española de Musicoterapeutas CAEMT, 2018, p. 2.https://d1784e03-2463-4a94-a09a8094b681dc16.filesusr.com/ugd/643546_6ef1ead252764666a6a176856266259f.pdf

Todos los documentos elaborados en el seno de las AEMTA-EMTC, anteriormente mencionados, contribuyen a dar "estructura a la profesión, aportan el marco de referencia organizativo y constituyen las bases organizativas necesarias para trabajar por el reconocimiento de la musicoterapia como profesión ante las autoridades competentes" (Sabbatella et al., 2018, p. 4). 
Por otro lado, muy cercano a las AEMTA-EMTC está la Asociación española de musicoterapeutas profesionales (AEMP), una organización sindical perteneciente al Ministerio de Trabajo y Asuntos Sociales. Se puede pertenecer a ella:

- De manera individual (si se está asociado a una asociación de Musicoterapia registrada en Ministerio de Interior).

- De manera colectiva (toda una asociación de Musicoterapia registrada en Ministerio de Interior).

Este sindicato está encargado de gestiones relacionadas con el reconocimiento de la profesión. Dentro de AEMP, se creó la CAEMT, que gestiona el REMTA, cuyos detalles se explicaron anteriormente (AEMP, 2021b).

De más reciente creación es la Federación Española de Asociaciones de Musicoterapia FEAMT, que merece un espacio aparte en cuanto al trabajo por la unión de musicoterapeutas en el camino de la regulación profesional de la Musicoterapia. Yendo a los orígenes, y después de diversos intentos infructuosos en 1999-2000, se empieza a gestar un nuevo intento por configurar una Federación. El Dr. Alejandro March Luján, por aquel entonces presidente de la AVMT, después de varios contactos con las presidencias de varias asociaciones nacionales y con la organización del "II Congreso de Terapias creativas" que se iba a celebrar en Vitoria (País Vasco), lanzó una convocatoria abierta vía mail a todas las asociaciones españolas de Musicoterapia de las que se tenía constancia en aquel momento, para tener así una primera reunión. Aprovechando el amparo del congreso anteriormente mencionado, en julio de 2013 se realiza este primer encuentro para establecer las bases de la Federación ${ }^{29}$. Después de éste y otros 2 encuentros más (diciembre 2013 y marzo 2014) con asociaciones que habían acudido a la convocatoria ${ }^{30}$, quedó aprobada definitivamente la FEAMT en julio

\footnotetext{
${ }^{29}$ A esta $1^{\text {a }}$ convocatoria asistieron: por AVMT, Alejandro March como presidente; por APM, Manuel Sequera como vicepresidente y Carmen Miranda como representante; por ADIMTE, Sara Añino Villalva como presidenta; por MAP, Carmen Angulo; por el Instituto Europeo de Musicoterapia Humanista IEMH, Lucía Díaz Uceda como presidenta; y por ACLEDIMA, $\mathrm{M}^{\mathrm{a}}$ Teresa del Moral Marcos como representante.

${ }^{30}$ A estas reuniones asistieron AVMT, ADIMTE, la Asociación para el estudio e investigación de la Música, la Terapia y la Comunicación (AEIMTC), el Instituto Europeo de Musicoterapia Humanista (IEMH), la Asociación Española de Musicoterapia Plurimodal (AEMtP) y AGAMUT, participando como asociaciones observadoras la ACMT y la APM (que finalmente no llegaron a formar parte de la FEAMT por decisión propia).
} 
de 2014 con el $n^{\circ} 51190$ en el Registro Nacional de Asociaciones del Ministerio de Interior (FEAMT, 2021a). El primer presidente de la FEAMT fue el Dr. Alejandro March Luján (de julio 2014 a julio 2017), pasando el testigo a la musicoterapeuta $\mathrm{D}^{\mathrm{a}}$ Guacimara Molina (julio 2017-julio 2020), y siendo la actual presidenta la

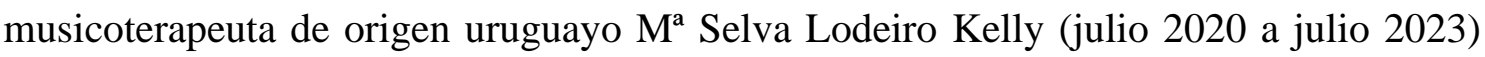
(March-Luján, 2021).

Las asociaciones que componen actualmente la FEAMT son (por orden alfabético y concretando información de aquellas que no se detallaron en el apartado anterior):

1. Asociación Balear de Musicoterapeutas ABAMU(con sede en Mallorca, en la comunidad de Illes Balears). Su actual presidenta es la musicoterapeuta de origen argentino $\mathrm{D}^{\mathrm{a}}$ Gabriela Guaglione.

2. ADIMTE.

3. AEIMTC (con sede ubicada en Vitoria, en el País Vasco).Su actual presidenta es la musicoterapeuta $\mathrm{D}^{\mathrm{a}}$ Jaione Oribe.

4. AEMtP(son sede ubicada en Valencia). Su actual presidenta es la musicoterapeuta de origen uruguayo $\mathrm{D}^{\mathrm{a}} \mathrm{M}^{\mathrm{a}}$ Selva Lodeiro Kelly.

5. Asociación para la evolución de la Musicoterapia en Canarias AEMUCAN (con sede el Gran Canaria, en la comunidad autónoma de Canarias).Su actual presidenta es la musicoterapeuta $\mathrm{D}^{\mathrm{a}}$ Guacimara Molina Sosa.

6. AGAMUT.

7. Asociación de Musicoterapia de Andalucía AMA (con sede en Sevilla, en la Comunidad autónoma de Andalucía). Su actual presidenta es la musicoterapeuta D ${ }^{\mathrm{a}}$ Lourdes Carmona.

8. Asociación de Musicoterapia Región de Murcia AMTRM. Su actual presidenta es la musicoterapeuta es Da Raquel López Gómez.

9. Asociación de profesionales de la Musicoterapia en Extremadura APROMTEX (con sede en Badajoz, en la Comunidad autónoma de Extremadura). Su actual presidenta es la musicoterapeuta $\mathrm{D}^{\mathrm{a}}$ Silvia Rufo.

10. AVMT. (FEAMT, 2021b) 
La FEAMT trabaja creando redes entre musicoterapeutas y promoviendo la calidad en la práctica profesional contribuyendo a la visibilización y divulgación de la musicoterapia como disciplina terapéutica. Para ello, mantiene reuniones periódicas de gestión y la coordinación de comisiones de trabajo que elaboran documentos orientativos para el buen desarrollo ético y profesional de la musicoterapia. En este sentido, la FEAMT ha venido refrendando documentos elaborados y aprobados en el seno de las AEMTA EMTC, y confeccionando otros nuevos, a saber:

\begin{tabular}{|c|c|}
\hline $\begin{array}{l}\text { Documentos aprobados en seno de AEMTA- } \\
\text { EMTC }^{31} \text { refrendados por FEAMT }\end{array}$ & Documentos originales elaborados por FEAMT \\
\hline 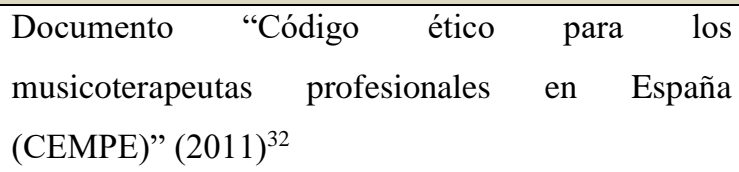 & $\begin{array}{l}\text { Documento "Perfil profesional del } \\
\text { musicoterapeuta" } 33\end{array}$ \\
\hline $\begin{array}{l}\text { Documento "Criterios para ser musicoterapeuta } \\
\text { profesional en España" }(2009)^{34}\end{array}$ & $\begin{array}{l}\text { Documento recopilatorio "Acciones llevadas a } \\
\text { cabo tanto por la Federación Española de } \\
\text { Asociaciones de Musicoterapia (FEAMT) como } \\
\text { por algunas de las asociaciones que la integran, con } \\
\text { el propósito de contribuir a la regulación } \\
\text { profesional"35 }\end{array}$ \\
\hline $\begin{array}{lccc}\text { Documento } & \text { Técnico } & \text { "Recomendaciones } \\
\text { Orientativas para Valorar el Diseño de los } \\
\text { Postgrados Universitarios de } \text { Musicoterapia en } \\
\text { España" (diciembre 2008) }{ }^{36} \text {. }\end{array}$ & $\begin{array}{l}\text { Documento "Criterios y proceso que la FEAMT } \\
\text { recomienda, e insta a seguir a las asociaciones } \\
\text { federadas, para la valoración de las solicitudes de } \\
\text { socios que provengan de musicoterapeutas } \\
\text { egresados de formaciones no presenciales" } 37\end{array}$ \\
\hline
\end{tabular}

\footnotetext{
${ }^{31}$ Todos los documentos que se aprecian en esta columna fueron aprobados originariamente en seno de AEMTA-EMTC en la fecha que se indica en cada uno, y posteriormente fueron refrendados en asamblea de FEAMT por decisión unánime el 29 de abril de 2016.

${ }^{32}$ Disponible en http://feamt.es/wp-content/uploads/2020/11/CODIGO-ETICO-FEAMT-.pdf

${ }^{33}$ Aprobado unánimemente en asamblea FEAMT en julio de 2017. Disponible en http://feamt.es/wpcontent/uploads/2020/04/PERFIL-PROFESIONAL-DEL-MUSICOTERAPEUTA.pdf

34 Disponible en http://feamt.es/wp-content/uploads/2021/02/Criterios-para-ser-MT-en-Es. pa\%C3\%B1a.pdf

${ }_{35}$ Aprobado unánimemente en asamblea FEAMT en octubre de 2018. Disponible en http://feamt.es/wpcontent/uploads/2020/04/HISTORICO-DE-ACCIONES-REGULACION-PROFESIONAL.pdf 36 Disponible en https://d1784e03-2463-4a94-a09a8094b681dc16.filesusr.com/ugd/643546 c042420329814b7c817eeff67a103769.pdf

37 Estos criterios fueron aprobados en asamblea FEAMT en febrero de 2020. Disponible en http://feamt.es/wp-content/uploads/2020/04/CRITERIOS-Y-REQUISITOS-FEAMT-PARA-

FORMACIONES-NO-PRESENCIALES.pdf
} 


\begin{tabular}{|l|l|l|}
\hline $\begin{array}{l}\text { Registro Español de Musicoterapeutas Acreditados } \\
(\text { REMTA, 2010 })^{38}\end{array}$ & $\begin{array}{l}\text { Comunicado "Reflexiones sobre el ejercicio } \\
\text { profesional durante el estado de alarma por } \\
\text { COVID-19"39 }\end{array}$ \\
\hline $\begin{array}{l}\text { Sistema Nacional de Acreditación de } \\
\text { Musicoterapeutas - Reglamento de Funcionamiento } \\
\text { Interno (RFI) de la Comisión de Acreditación } \\
\text { Española de Musicoterapeutas Profesionales } \\
(\text { CAEMT, noviembre 2010) }\end{array}$ & \\
\hline $\begin{array}{l}\text { Criterios para la Organización de } \\
\text { Congresos Nacionales de Musicoterapia }\end{array}$ & \\
$(2015)$ & & \\
\hline
\end{tabular}

Tabla 3 Documentos aprobados o refrendados desde FEAMT.

Nota. El criterio de aparición de los documentos en esta tabla se ha hecho por orden cronológico en cada columna, de más antiguos a más modernos. Adaptado de "Documentos", por FEAMT, 2021c.http://feamt.es/documentos/

Encuentros y desencuentros políticos.

En la búsqueda de la regulación profesional, se hace necesario reseñar las acciones promovidas por algunas asociaciones de ámbito regional en distintas Comunidades Autónomas. La idea conjunta de las asociaciones era orquestar una maniobra desde los parlamentos regionales para que desde estas instituciones llegará una propuesta firme multifocalizada al Gobierno Central, para así tener una mayor fuerza de cara a la pretendida regulación de la Musicoterapia.

La $1^{\text {a }}$ en materializar este tipo de iniciativas fue $\operatorname{AMTRM}^{41}$ (de la mano de su presidente en aquel momento, el Dr. Alfonso López Ruiz) desde el pleno de la Asamblea Regional de Murcia. El día 3 de febrero de 2017, a través del Grupo Parlamentario Popular, presentó una moción sobre la regulación del trabajo de los musicoterapeutas. Mediante dicha moción, la Asamblea Regional de Murcia instó al

\footnotetext{
${ }^{38}$ Más información disponible en https://musicoterapeutas.wixsite.com/aemp/remta

${ }^{39}$ Aprobado unánimemente en asamblea FEAMT en abril de 2020. Disponible en http://feamt.es/wpcontent/uploads/2020/05/Comunicado-abril-2020_.pdf

${ }^{40}$ Disponible en https://www.geyseco.es/musicoterapia/documentos/Libro_Actas.pdf, anexo 6, pp. 228237
} 
consejo de Gobierno para que éste a su vez inste al Gobierno de la Nación a regula los requisitos que debe cumplir el musicoterapeuta para desarrollar su trabajo y los requisitos que debe cumplir el musicoterapeuta para desarrollar su trabajo en la región de Murcia (FEAMT, 2021d).Lamentablemente, esta iniciativa quedó pendiente de aprobación, pero sirvió como modelo para la AVMT, que se detallará a continuación.

Por su parte, la AVMT (de la mano de su presidente en aquel momento, D. David Buedo Salas), el 14 de marzo de 2018, gracias al contacto que tuvieron los parlamentarios del Grupo Popular que habían impulsado la iniciativa murciana con los homónimos valencianos, presentó ante la Comisión de Política Social y de Trabajo del Parlamento Valenciano, una PNL (Proposición no de Ley) ${ }^{42}$ de tramitación ordinaria, sobre el impulso y regulación de la Musicoterapia, la cual fue aprobada por unanimidad. La PNL aprobada instaba al Consejo Valenciano para que:

1. este a su vez inste al Gobierno de España a regular los requisitos académicos, laborales y de capacitación profesional que debe reunir el musicoterapeuta para poder ejercer adecuadamente y con plenas garantías esta profesión en el ámbito nacional, teniendo en cuenta las consideraciones de la FEAMT.

2. En los ámbitos de su competencia disponga, supervise y controle los requerimientos, y niveles de calidad, que ha de tener un musicoterapeuta para poder ejercer su trabajo en la Comunitat Valenciana, previniendo y evitando el intrusismo profesional.

3. Reconozca los efectos positivos de la musicoterapia en ámbitos sanitarios, educativos o sociales, incluyendo finalidades preventivas, dándole un impulso decidido para favorecer su desarrollo y consolidación en el ámbito de la Comunitat Valenciana.

4. Contribuya a difundir en qué consiste la musicoterapia, así como sus efectos benéficos, entre los responsables de la planificación sanitaria, educativa o de bienestar social, entre los centros, instituciones o entidades dedicados a dichos ámbitos, y entre la población en general.

\footnotetext{
${ }^{42}$ En España, PNL son unas siglas que significan "Proposición no de Ley", y hacen referencia a las iniciativas surgidas en las Cortes (Parlamentos Autonómicos Regionales) cuya finalidad sea la aprobación por estas de textos o resoluciones que no tengan carácter de ley. Suponen una especie de "declaración de intenciones" políticas que instan a los órganos competentes del Gobierno Central a tomar medidas en base a lo expuesto en estos textos.
} 
5. Elabore, en colaboración con la Asociación Valenciana de Musicoterapia, un plan de fomento de la musicoterapia en la Comunitat Valenciana, de carácter transversal, que contemple su utilidad como herramienta social.

6. Subscriba convenios para el desarrollo de actividades musicoterapéuticas en los ámbitos clínicos, docentes, investigadores. (FEAMT, 2021d)

Si bien esta PNL indicaba que deberían llevarse a cabo las acciones referidas al Consejo Valenciano en un plazo de seis meses, por diferentes circunstancias, no llegaron a término. De igual manera, tampoco se llevaron a cabo las que esta comisión instaba al Gobierno Central. Por tanto, esta PNL se quedó en un conjunto de medidas propuestas sin continuidad (al menos, por el momento).

Casi de manera paralela al movimiento que realizó la AVMT y a través del Grupo Parlamentario Popular, ABAMU presenta ante la Comisión de Política Social y Empleo de del Govern Balear, una PNL (Proposición no de Ley) de tramitación con carácter de urgencia, sobre el impulso y regulación de la Musicoterapia, la cual fue aprobada por unanimidad el 8 de mayo de 2018. El texto de dicha PNL era prácticamente el mismo que el indicado en el caso de la AVMT, pues el contacto con la AVMT fue en todo momento muy fluido, replicando los pasos anteriores de este lado (FEAMT, 2021d). Por causas desconocidas, las demandas de esta PNL en les Illes Balears aún no se han llevado a la práctica.

De manera similar a las anteriores, y en fechas muy cercanas a las mismas, el Partido Aragonés (PAR), dentro de la Comunidad de Aragón, también presentó una PNL ante La Comisión de Innovación, Investigación y Universidad en sesión celebrada el día 27 de septiembre de 2018, para que el Gobierno de Aragón iniciase las gestiones necesarias para regular la Musicoterapia, y promover su conocimiento y utilización en beneficio de las personas. Esta PNL fue aprobada por unanimidad en la fecha indicada anteriormente, y publicada en el Boletín Oficial de las Cortes de Aragón el 2 de octubre del mismo año. (Cortes de Aragón, 2018)

También merecen ser reseñados los encuentros que, bien desde el seno de la FEAMT o por iniciativas musicoterapeuticas, se han tenido entre representantes de la Musicoterapia con altos cargos de la Administración o la Política en el ámbito estatal. En este sentido, se señalarán algunos de las más importantes: 
- Director General de Ordenación Profesional, Ministerio de Sanidad, 9 junio 2015. Surgió la posibilidad de tener este encuentro, al que acudieron representantes de la FEAMT: su presidente (Dr. Alejandro March Luján) y secretaria (Carmen Angulo Sánchez-Prieto) en aquel entonces. En ella quedó patente, desde la dirección general, la imposibilidad de que la Musicoterapia fuese regulada como profesión sanitaria, pué son estaba incluida en el catálogo de estas profesiones. Por tanto, el encuentro fue infructuoso desde la finalidad, pero valorable como presentación de la Musicoterapia ante la Administración.

- Reunión con el Ministerio de Sanidad, 22 octubre de 2020. Esta reunión fue buscada y organizada por la entidad privada Huella Sonora Musicoterapia, que convocó a personas con representatividad en Musicoterapia (que se presentó como Grupo de Trabajo para la Regulación de la Musicoterapia en España) en el Estado Español. Los asistentes a la reunión fueron:

- Por el Ministerio de Sanidad: Rodrigo Gutiérrez, médico y director General de ordenación profesional en el Ministerio de Sanidad; $\mathrm{M}^{\mathrm{a}}$ Ángeles López Oribe, subdirectora General de ordenación profesional en el Ministerio de Sanidad, responsable de la regulación de medicina preventiva; y Yolanda Álvarez González, vocal asesora de la dirección General, como jurista.

- Grupo de Trabajo para la Regulación de la Musicoterapia en España: Carmen Angulo Sánchez Prieto, Alejandro March Luján, Guacimara MolinaSosa, Carmen Miranda Pereda, Marta Nieto Gavilán, y Manuel Sequera Martín.

En este encuentro se pudieron exponer los argumentos para que la Musicoterapia pasara a considerarse como profesión sanitaria (como sucede en otros países), y poder iniciar así los trámites necesarios desde la Administración. Los representantes del Ministerio expusieron que los pasos que se venían realizando en España eran positivos (proceso REMTA, formación universitaria asentada en formato de máster-postgrado, evidencia científica creciente), pero insistieron en que la vía inmediata para estar en el camino pretendido era conseguir cuanto antes la conversión de la formación en Musicoterapia en un formato más extenso, en formato de grado universitario.

Pero en la senda hacia la Regulación Profesional, también es importante reseñar algunos sucesos recientes que han puesto en entredicho la validez de la Musicoterapia, 
con especial impacto debido la fuente de origen. En este sentido, en noviembre de 2018, el Ministerio de Sanidad, Consumo y Bienestar Social"3, elaboraron el "Plan para la protección de la salud frente a las pseudoterapias"44. A raíz de este plan, salió a la luz un primer borrador/ informe preliminar a modo de listado de terapias, detallando que:

El análisis exploratorio realizado indica que para la mayoría de las técnicas con pretendida finalidad sanitaria analizadas (73 de las 139 incluidas) no se han identificado publicaciones científicas con diseños que permitan establecer su eficacia de forma robusta (ensayos clínicos ni revisiones sistemáticas o metaanálisis publicados en Pubmed) durante el periodo 2012-2018. Es decir, más de la mitad, no tienen soporte en el conocimiento científico con metodología lo suficientemente sólida que sirva para evaluar su seguridad, efectividad y eficacia (Ministerio de Sanidad, Consumo y Bienestar Social; Ministerio de Ciencia, Innovación y Universidades; Instituto de Salud Carlos III; 2018, p.1)

En este sentido, cabe destacar que la Musicoterapia cuenta con publicaciones científicas en esta base de datos con esos criterios indicados, aunque de momento está incluida en este informe dentro del bloque "Terapias aún en evaluación” (un total de 66), una suerte de semáforo amarillo que deja a nuestra disciplina en un limbo. En este tiempo en el que se está saliendo de la pandemia por Covid 19, los organismos competentes designados por el Ministerio han podido contactar con los órganos representativos de la Musicoterapia (FEAMT, AEMTA-EMTC, AEMP) y se está trabajando por poder aportar la evidencia necesaria para poder salir de este poco deseable catálogo.

Jornadas y Congresos de Musicoterapia

Siguiendo con las acciones que aportan en el camino hacia la regulación profesional de la Musicoterapia en España, es indispensable mencionar las variadas reuniones científicas, jornadas y congresos, que periódicamente se realizan en nuestro país.

\footnotetext{
${ }^{43}$ Junto con el Ministerio de Ciencia, Innovación y Universidades; en colaboración con Instituto de Salud Carlos III.

${ }^{44}$ Puede consultarse una información más detallada en https://www.conprueba.es
} 
Respecto a las jornadas de musicoterapia, cabe decir que son eventos con un marcado carácter divulgativo, y algunas de ellas vienen realizándose desde hace décadas; en ellas se abordan temas específicos que suponen impulso en la profesión. Cabe destacar entre ellas las que siguen:

- "Jornadas Estivales de Musicoterapia" ${ }^{45}$, celebradas en Extremadura (V edición);

- "Jornadas de musicoterapia por la Salud y la educación" 46 organizadas por la “Asociación Valenciana de Musicoterapia AVMT” (XVI edición);

- Curso de Verano del "Centro de Investigación Musicoterapeutica CIM" de Bilbao (XXXII edición);

- "Escuela de Verano de Musicoterapia y Terapias Creativas" ${ }^{47}$ del Instituto “Música, Arte y Proceso MAP”, en Vitoria (XXXV edición) (March-Lujá, 2021).

Cabe también mencionar las "I Jornadas Hispanolusas de actualización en investigación musicoterapéutica" 48 celebradas en Valencia en 2016, con una representación de los musicoterapeutas investigadores punteros de España y Portugal. Este evento supuso un hito importante en el desarrollo de la investigación en Musicoterapia en la Península Ibérica. Asimismo, el colectivo musicoterapeuta en España, a través de la AEMTA-EMTC, también organiza los Congresos Nacionales de Musicoterapia. El I Congreso Nacional de Musicoterapia se celebró en el año 2006, en Barcelona. Desde entonces, los encuentros profesionales se han sucedido, reuniendo cada vez a más musicoterapeutas en pro de la calidad en la profesión, en esta sucesión:

- $\quad$ I Congreso Nacional de Musicoterapia, Montserrat (Barcelona), 2006.

- $\quad$ II Congreso Nacional de Musicoterapia, Zaragoza, 2008.

- $\quad$ III Congreso Nacional de Musicoterapia, Cádiz, 2010.

- IV Congreso Nacional de Musicoterapia, celebrado en Madrid, 2012.

- V Congreso Nacional de Musicoterapia, Barcelona 2014.

\footnotetext{
${ }^{45} \mathrm{https}$ ://jornadasestivalesmusicoterapia.wordpress.com/

${ }^{46}$ https://musicoterapiavalencia.org/jornadas

${ }^{47}$ https://www.institutomapvitoria.com/imap/35-escuela-de-verano

${ }^{48}$ http://jornadasinvestigacion.musicoterapiavalencia.org/
} 
- VI Congreso Nacional de Musicoterapia, Santiago de Compostela 2017.

- El último, VII Congreso Nacional de Musicoterapia, organizado por ADIMTE, fue celebrado en octubre del 2019, y reunió a 400 participantes en el Campus María Zambrano, en Segovia (March-Luján, 2021).

Además, España ha sido sede de Congresos de Musicoterapia de carácter internacional, de entre los que se destacan los siguientes:

- 1993: VII Congreso Mundial en Vitoria (España).

- 2010: VIII Congreso Europeo Musicoterapia en Cádiz, 5-9 mayo.

- 2017: III Congreso Iberoamericano de Investigación en Musicoterapia" Musicoterapia, Música y nuevos paradigmas en Salud" Valencia (España), 10 al 12 de noviembre (March-Luján, 2021).

También merece ser destacada la presencia creciente de musicoterapeutas con sus trabajos científicos en congresos de diversas sociedades científicas en ámbitos como psicología, cuidados paliativos, geriatría, atención hospitalaria, diversidad funcional, salud mental atención temprana, entre otras. Todo ello contribuye a la visibilidad de la musicoterapia y la creciente consideración de la disciplina por otros profesionales.

\section{Conclusión}

La disciplina de la Musicoterapia en España ha tenido una evolución paulatina en sus 50 años de vida. Si bien el presente formativo está consolidado en las formaciones de máster, el futuro parece estar abocado a un crecimiento en forma de grado universitario. En este sentido, la apuesta universitaria cobra especial relevancia. Las entidades representativas de la Musicoterapia (AEMTA-EMTC y FEAMT mayormente) están convocadas, junto con los órganos competentes de la Administración y Política Españolas, a ser agentes activos en el proceso hacia la Regulación de la profesión, que se antoja cercano e inexorable, y que debe mirarse en espejos de países en los que se ha logrado esta pretendida regulación. 
Agradecimientos: Especial gratitud a la comunidad Musicoterapêtica amiga, que brindaron información valiosa al autor del presente artículo tras conversaciones personales, en especial de la parte profesional actual por comunidades. Por orden alfabético: Ana Alegre, Carmen Angulo, Isabel Bellver, Eloisa Beltrán, Laura Cañellas, Jorge Cavia, Antonia de la Torre, Miguel Ángel Diví, Esther García, Pau Gimeno, Gabriela Guaglione, Cristina López, Raquel López, Carmen Miranda, Guacimara Molina, Marta Nieto , Diego Salamanca, Manuel Sequera

\section{REFERENCIAS}

Asociación Española de Musicoterapeutas AEMP (2021a).Registro Español de Musicoterapeutas Acreditados

(REMTA). https://musicoterapeutas.wixsite.com/aemp/remta

Asociación Española de Musicoterapeutas AEMP (2021b).Qué es AEMP. https://musicoterapeutas.wixsite.com/aemp/untitled

Cortes de Aragón (2018). Aprobación por la Comisión de Innovación, Investigación y Universidad de la Proposición no de Ley núm. 141/18, sobre el reconocimiento y regulación de la musicoterapia. Boletín Oficial de las Cortes de Aragón, Número 276,Año XXXVI, Legislatura IX, 2 de octubre de 2018, p. 2040.http://bases.cortesaragon.es/bases/original.nsf/(BOCA1)/EB9915DDDFFD BD80C125831B005113B0/\$File/BOCA_276.pdf?OpenElement

Escudé, N., \&Acanfora, F. (2018). Music and Medicine in Spain: History and New Developments of a Growing Discipline, 10(1), 45-47. https://doi.org/10.47513/mmd.v10i1.600

European Music Therapy Confederation EMTC (2021). Music Therapy in Spain. History and background.https://www.emtc-eu.com/spain

Federación Española de Asociaciones de Musicoterapia FEAMT (2021a). Historia. http://feamt.es/historia/

Federación Española de Asociaciones de Musicoterapia FEAMT (2021b). ¿Quiénes somos? http://feamt.es/

Federación Española de Asociaciones de Musicoterapia FEAMT (2021c). Documentos. http://feamt.es/documentos/

Federación Española de Asociaciones de Musicoterapia FEAMT (2021d). Acciones llevadas a cabo tanto por la Federación Española de Asociaciones deMusicoterapia (FEAMT) como por algunas de las asociaciones que la integran, conel propósito de contribuir a la regulación profesional.http://feamt.es/wp-content/uploads/2020/04/HISTORICO-DEACCIONES-REGULACION-PROFESIONAL.pdf

Grupo de Trabajo para la Regulación de la Musicoterapia en España (2020). Documento base reunión Ministerio de Sanidad de 22 octubre. Material no publicado.

March-Luján, V.A. (2021). Historia reciente de la Musicoterapia en España. Máster en Musicoterapia Universidad Miguel de Cervantes-Musicaycolor. Material no publicado.

Ministerio de Sanidad, Consumo y Bienestar Social; Ministerio de Ciencia, Innovación y Universidades; Instituto de Salud Carlos III (2018). CoNprueba, nueva campaña en ciencia y salud del Gobierno de España y forma parte del Plan contra las pseudoterapias y las pseudociencias.https://www.conprueba.es/ 
ORDEN 12/2013, de 14 de marzo, de la Conselleria de Educación, Cultura y Deporte, por la que se fijan los criterios para la determinación de las relaciones de puestos de trabajo y se publican las plantillas tipo de las escuelas infantiles (segundo ciclo), colegios públicos de Educación Primaria, colegios públicos de Educación Infantil y Primaria y centros de educación especial de titularidad de la

https://dogv.gva.es/datos/2013/03/21/pdf/2013_2775.pdf

Sabbatella, P. (2004). Music Therapy in Spain. Voices: A World Forum for Music Therapy.http://www.voices.no/country/monthspain_march2004.html.

Sabbatella, P. L., del Moral, M. T., y Mercadal-Brotons, M. (2018). Perspectiva Contemporánea de la Musicoterapia en España (2000-2018). SONOGRAMA Magazine, Revista de pensament musical $i$ difusió cultural, 38. https://sonograma.org/2018/04/perspectiva-contemporanea-de-la-musicoterapiaen-espana/ 